\title{
PRIMES DIVIDING CHARACTER DEGREES AND CHARACTER ORBIT SIZES
}

\author{
DAVID GLUCK
}

\begin{abstract}
We consider an abelian group $A$ which acts faithfully and coprimely on a solvable group $G$. We show that some $A$-orbit on $\operatorname{Irr}(G)$ must have cardinality divisible by almost half the primes in $\pi(A)$. As a corollary, we improve a recent result of $\mathrm{I}$. M. Isaacs concerning the maximum number of primes dividing any one character degree of a solvable group.
\end{abstract}

A recent result of I. M. Isaacs [5, Corollary 4.3] relates the maximum number of primes dividing any one irreducible character degree of a solvable group $G$ to the number of primes dividing all the character degrees of $G$ taken together. Here we considerably strengthen the bound in [5, Corollary 4.3] by proving a result on character orbit sizes in coprime actions.

We consider an abelian group $A$ which acts faithfully and coprimely on a solvable group $G$. We show that some $A$-orbit on $\operatorname{Irr}(G)$ must have cardinality divisible by almost half the primes in $\pi(A)$. Our approach roughly parallels that of [6]. This paper and [5] contain new applications of the results and methods of $[6,7,1,2]$.

I would like to thank I. M. Isaacs for bringing this problem to my attention.

Our notation is largely standard. If a group $G$ acts on a set $\Omega$ and $\omega \in \Omega$, we denote by $\operatorname{Orb}_{G}(\omega)$ the $G$-orbit of $\omega$. If $G \triangleleft H$ and $H$ also acts on $\Omega$, we say that $h \in H$ moves $\operatorname{Orb}_{G}(\omega)$ if $\omega^{h} \notin \operatorname{Orb}_{G}(\omega)$. All groups considered in this paper are finite and solvable.

We now state our main results.

THEOREM 1. Let $A$ act faithfully on $G$ with $(|A|,|G|)=1$ and $A$ abelian. Then $|\pi(A)| \leqslant 2\left|\pi\left(\mathrm{Orb}_{A}(\chi)\right)\right|+8$ for some $\chi \in \operatorname{Irr}(G)$.

Corollary 1. Let $G$ be solvable. Let $s=\max \{|\pi(\chi(1))|: \chi \in \operatorname{Irr}(G)\}$ and let $\rho=\left|\pi\left(\Pi_{\chi \in \operatorname{Irr}(G)} \chi(1)\right)\right|$. Then $\rho \leqslant s^{2}+10 s$.

Proof. Using Theorem 1 above, we get a stronger version of [5, Theorem 4.1] in which part (a) is replaced by $|\rho(G)-\rho(N)-\sigma| \leqslant 2(s-|\sigma|)+8$. This leads to a stronger version of $\left[5\right.$, Corollary 4.3] in which $|\rho(G)| \leqslant s+\sum_{i=0}^{s-1}(2(s-i)+8)=s^{2}$ $+10 s$. Since $|\rho(G)|$ in [5] is called $\rho$ in our paper, this completes the proof.

Received by the editors November 13, 1985 and, in revised form, August 30, 1986. Presented on July 7 , 1986 to the A.M.S. Summer Conference on Representation Theory of Finite Groups, Arcata, California. 1980 Mathematics Subject Classification (1985 Revision). Primary $20 \mathrm{C} 15$. 
The next proposition shows that the bound in Theorem 1 is close to best possible.

Proposition 1. Let $m$ be a positive integer. There exist groups $A$ and $G$ satisfying the hypotheses of Theorem 1 with $|\pi(A)|=2 m$ and $\pi\left(\operatorname{Orb}_{A}(\chi)\right) \leqslant m$ for all $\chi \in \operatorname{Irr}(G)$.

Proof. For $1 \leqslant i \leqslant m$, choose odd primes $p_{i}$ and $q_{i}$ subject to the following conditions. Let $e_{i}$ and $f_{i}$ denote the order of $2 \bmod p_{i}$ and $q_{i}$ respectively.

(1) $\min \left(p_{i}, q_{i}\right)>2^{p_{i-1} q_{i-1}}$ for $i>1$,

(2) $q_{i} \equiv 2\left(\bmod e_{1} f_{1} e_{2} f_{2} \cdots e_{i-1} f_{i-1}\right)$ for $i>1$,

(3) $f_{i}<e_{i}$ for all $i$.

For each $i$ set $n_{i}=f_{i} p_{i}$. Clearly $2^{f_{i}}-1 \equiv 0 \bmod q_{i}$. Since

$$
\left(2^{n_{i}}-1\right) /\left(2^{f_{i}}-1\right) \equiv 1+2^{f_{i}}+\cdots+2^{\left(p_{i}-1\right) f_{i}},
$$

it follows that $\left(2^{n_{i}}-1\right) /\left(2^{f_{i}}-1\right) \equiv p_{i} \not \equiv 0 \bmod q_{i}$. By (3) above, $2^{n_{i}}-1=2^{f_{i} p_{i}}-1$ $\equiv 2^{f_{i}}-1 \not \equiv 0 \bmod p_{i}$.

Let $V_{i}$ be elementary abelian of order $2^{n_{i}}$. Let $C_{i}$ be cyclic of order $2^{n_{i}}-1$. Let $P_{i}$ be cyclic of order $p_{i}$ and let $P_{i}$ act on $C_{i} \cong \mathrm{GF}\left(2^{n_{i}}-1\right)^{\times}$as the subgroup of order $p_{i}$ in $\operatorname{Gal}\left(\mathrm{GF}\left(2^{n_{i}}\right) / \mathrm{GF}(2)\right)$. Let $H_{i}=\left(P_{i} C_{i}\right) V_{i}$ be the corresponding subgroup of the affine semilinear group over $\operatorname{GF}\left(2^{n_{i}}\right)$. Let $Q_{i}$ be the Sylow $q_{i}$-subgroup of $C_{i}$. The preceding paragraph implies that $\left[P_{i}, Q_{i}\right]=1$ and $Q_{i}>1$. Let $C_{i}=Q_{i} \times R_{i}$. Let $G_{i}=R_{i} V_{i}$ and $A_{i}=P_{i} Q_{i}$. Then $A_{i}$ acts faithfully on $G_{i}$ and the preceding paragraph shows that $\left(\left|A_{i}\right|,\left|G_{i}\right|\right)=1$.

Let $\chi \in \operatorname{Irr}\left(H_{i}\right)$ and let $\lambda$ be an irreducible constituent of $\chi_{V_{i}}$. If $\lambda=1$, then $\chi(1)$ divides $p_{i}$. If $\lambda \neq 1$, then $\left|I_{H_{i}}(\lambda): V_{i}\right|=p_{i}$, and every character in $\operatorname{Irr}\left(I_{H_{i}}(\lambda) \mid \lambda\right)$ has degree 1 (see $\left[4,6.17\right.$ and 6.20]). Hence $\chi(1)=\left|Q_{i}\right|\left|R_{i}\right|$. In either case, at most one prime in $\pi\left(A_{i}\right)$ divides $\chi(1)$.

Let $\psi \in \operatorname{Irr}\left(G_{i}\right)$. Since $G_{i} \triangleleft H_{i},\left|\mathrm{Orb}_{A_{i}}(\psi)\right|$ divides an irreducible character degree of $H_{i}$. Hence $\left|\pi\left(\mathrm{Orb}_{A_{i}}(\psi)\right)\right| \leqslant 1$.

Now let $G=G_{1} \times \cdots \times G_{m}$ and let $A=A_{1} \times \cdots \times A_{m}$ act componentwise on $G$. Clearly $A$ acts faithfully on $G$. To show that $(|A|,|G|)=1$, it suffices, by induction, to show that $\left(\left|A_{m}\right|,\left|G_{i}\right|\right)=\left(\left|A_{i}\right|,\left|G_{m}\right|\right)=1$ for $i \leqslant m$. We already know that $\left(\left|A_{m}\right|,\left|G_{m}\right|\right)=1$. Suppose $i<m$. If $\left(\left|A_{m}\right|,\left|G_{i}\right|\right)>1$, then $\left(\left|A_{m}\right|,\left|C_{i}\right|\right)=\left(\left|A_{m}\right|\right.$, $\left.2^{p_{i} f_{i}}-1\right)>1$. Since $f_{i}$ divides $q_{i}-1$, this contradicts condition (1) in the first paragraph. If $\left(\left|A_{i}\right|,\left|G_{m}\right|\right)>1$ for $i<m$, then $\left(\left|A_{i}\right|,\left|C_{m}\right|\right)=\left(\left|A_{i}\right|, 2^{p_{m} f_{m}}-1\right)>1$. Then ord ${ }_{r}(2) \mid p_{m} f_{m}$, where $r=p_{i}$ or $r=q_{i}$. Since ord ${ }_{r}(2)$ divides $r-1$ and $r<p_{m}$, we have ord ${ }_{r}(2) \mid f_{m}$ and so ord $r(2) \mid\left(q_{m}-1\right)$, contrary to condition (2) in the first paragraph. Hence $A$ and $G$ satisfy the hypothesis of Theorem 1 .

Let $\psi=\psi_{1} \times \cdots \times \psi_{m}$ be an arbitrary character in $\operatorname{Irr}(G)$. As above, we may choose $S_{i} \in\left\{P_{i}, Q_{i}\right\}$ so that $S_{i}$ fixes $\psi_{i}$. Then $S_{1} \times \cdots \times S_{m}$ fixes $\psi$, so $\left|\pi\left(\operatorname{Orb}_{A}(\psi)\right)\right| \leqslant m$. This completes the proof.

We proceed to prove Theorem 1. We will use the proof of [6, Theorem 3.3] as a rough guide in the proof of Proposition 3 below.

Lemma 1. Let $A$ act on $G$ with $A$ abelian and $(|A|,|G|)=1$. Let $A_{p}$ denote the p-Sylow subgroup of $A$. Let $N$ and $M$ be $A$-invariant normal subgroups of $G$, with $\left[A_{p}, G\right] \leqslant M$ and $N \leqslant M$. Let $\lambda$ be an irreducible character of $N$ and suppose $A_{p}$ 
moves $\operatorname{Orb}_{M}(\lambda)$. Then $A_{p}$ moves $\operatorname{Orb}_{G}(\lambda)$. Similarly, if $v \in N$ and $A_{p}$ moves $\operatorname{Orb}_{M}(v)$, then $A_{p}$ moves $\operatorname{Orb}_{G}(v)$.

Proof. Suppose $A_{p}$ stabilizes $\operatorname{Orb}_{G}(\lambda)$. Then the semidirect product $A_{p} G$ acts on $\operatorname{Orb}_{G}(\lambda)$ with $G$ acting transitively. By Glauberman's Lemma [4, Lemma 13.8], $A_{p}$ fixes some $\psi \in \operatorname{Orb}_{G}(\lambda)$. Then $A_{p}^{g}$ fixes $\lambda$ for some $g \in G$. Since $G=$ $C_{G}\left(A_{p}\right)\left[A_{p}, G\right]=C_{G}\left(A_{p}\right) M$, we may assume that $g \in M$. Hence $A_{p}$ stabilizes $\operatorname{Orb}_{M}(\lambda)$.

The second assertion is proved similarly.

LEMma 2. Let $G \neq 1$ be solvable with every normal abelian subgroup cyclic. Let $p_{1}, \ldots, p_{n}$ be the distinct prime divisors of $|F(G)|$ and let $Z \leqslant Z(F(G)$ ) with $|Z|=p_{1} \cdots p_{n}$. Let $D=C_{G}(Z)$. Then there exist $E, T \triangleleft G$ with

(i) $E T=F(G)$ and $E \cap T=Z$.

(ii) Each Sylow subgroup of $T$ is cyclic, dihedral, semidihedral or quaternion.

(iii) $T$ has a cyclic subgroup $U$ with $|T: U| \leqslant 2$ and $U \triangleleft G$.

(iv) Each Sylow subgroup of $E$ is cyclic of prime order or extraspecial of prime exponent or exponent 4.

(v) $G$ is nilpotent if and only if $G=T$.

(vi) $T=C_{G}(E)$ and $F(G)=C_{D}(E / Z)$.

(vii) Each Sylow subgroup of $E / Z$ is elementary abelian and is a completely reducible $D / F(G)$-module.

Proof. This is [7, Corollary 2.4].

Lemma 3. Let $G, E, U$, and $Z$ be as in Lemma 2. Let $V$ be a faithful $F[E U]$-module for a finite field $F$. Let $W \neq 0$ be an irreducible $U$-submodule of $V$ and let $e=|E: Z|^{1 / 2}$. Then $\operatorname{dim} V=m e \operatorname{dim} W$ for an integer $m$.

Proof. This is [7, Lemma 2.5].

Lemma 4. Let $E \triangleleft H$ with $|H: E|=p$ and $p+|E|$. Let $Z=Z(E), P \in \operatorname{Syl}_{p}(H)$, and let $V$ be a finite-dimensional $F[H]$-module for a field $F$. Assume that $E / Z$ is an abelian q-group for a prime $q, P \nless C_{H}(E)$, and $V_{E}$ is a faithful, completely reducible and homogeneous module. Then $\operatorname{dim} C_{V}(P) \leqslant(\operatorname{dim} V) / 2$ if $p$ is odd.

Proof. This is part of [6, Lemma 1.7].

LEMmA 5. Let $V \neq 0$ be a faithful and completely reducible $F[G]$-module for a field $F$ and a solvable group $G$. Then $|G| \leqslant|V|^{9 / 4}$.

Proof. This is a slightly weaker version of [7, Theorem 3.1].

Proposition 2. Let $A$ act on $G$ with $(|A|,|G|)=1$ and $A$ cyclic of squarefree order. Suppose that $\left[A_{p}, G / F(G)\right]$ is a nonidentity abelian growip for all $p$ in $\pi(A)$. Then $A$ has a faithful orbit on $\operatorname{Irr}(G)$.

Proof. Let $H=G / F(G)$. Then $[A, H]=\prod_{p \in \pi(A)}\left[A_{p}, H\right]$ is contained in $F(H)$. Let $W=[A, H] / \Phi([A, H])$, so that $W$ is a direct product of elementary abelian $q$-groups for primes $q$ dividing $|H|$. Write $W=W_{1} \times \cdots \times W_{k}$, each $W_{i}$ an irreducible $A$-module. For $1 \leqslant i \leqslant k$, let $1 \neq \lambda_{i} \in \operatorname{Irr}\left(W_{i}\right)$. Let $\lambda=\lambda_{1} \times \cdots \times \lambda_{k}$. Since 
$(|A|,|H|)=1, A$ acts faithfully on $[A, H]$ and hence on $W$. For each $i, W_{i}$ is a faithful irreducible module for the cyclic group $A / C_{A}\left(W_{i}\right)$. Hence $\lambda_{i}$ is moved by $A_{p}$ for every $p \in \pi\left(A / C_{A}\left(W_{i}\right)\right)$. Thus $\lambda$ lies in a faithful $A$-orbit on $\operatorname{Irr}(W) \leqslant$ $\operatorname{Irr}([A, H])$. We now apply Lemma 1 with $A, H,[A, H],[A, H]$ in place of $A, G, M, N$ and conclude that $\operatorname{Orb}_{G}(\lambda)$ is moved by $A_{p}$ for every $p \in \pi(A)$. Let $\chi \in \operatorname{Irr}(H \mid \lambda)$. Then $\chi$ lies in a faithful $A$-orbit on $\operatorname{Irr}(H) \leqslant \operatorname{Irr}(G)$.

Proposition 3. Let $\pi_{0}=\{2,3,5,7,11,13,17,31\}$. Let $A$ be cyclic of squarefree $\pi_{0}^{\prime}$-order. Let $A$ act on $G$ with $(|A|,|G|)=1$ and $\left[A_{p}, G\right]$ nonabelian for all $p \in \pi(A)$. Let $V$ be an abelian group which is a direct product of completely reducible AG-modules over various finite fields. Suppose $(|A|,|V|)=1$ and $A G$ acts faithfully on $V$. Then there exists $v \in V$ such that $\operatorname{Orb}_{G}(v)$ is moved by every $A_{p}$.

Proof. We proceed by induction on $|G|+|V|$. Set $\pi(A)=\pi$.

First suppose $V$ is not an irreducible $A G$-module. Write $V=V_{1} \times \cdots \times V_{k}$, with each $V_{i}$ an irreducible $A G$-module. Let $\bar{G}_{i}=G / C_{G}\left(V_{i}\right)$ for $1 \leqslant i \leqslant k$. Let $\pi_{i}=\{p$ $\in \pi:\left[A_{p}, \bar{G}_{i}\right]$ is nonabelian $\}$. For each $p \in \pi,\left[A_{p}, G\right]$ is isomorphic to a subgroup of $\left[A_{p}, \bar{G}_{1}\right] \times \cdots \times\left[A_{p}, \bar{G}_{k}\right]$. Hence $\left[A_{p}, \bar{G}_{i}\right]$ is nonabelian for some $i$, and so $\pi=\pi_{1} \cup \cdots \cup \pi_{k}$. We apply the induction hypothesis to $\bar{G}_{i}$ with $\prod_{p \in \pi_{i}} A_{p}, \bar{G}_{i}, V_{i}$ in place of $A, G, V$. We obtain $v_{i} \in V_{i}$ such that $\operatorname{Orb}_{G}\left(v_{i}\right)$ is moved by $A_{p}$ for all $p \in \pi_{i}$. Then $\operatorname{Orb}_{G}\left(v_{1}, \ldots, v_{k}\right)$ is moved by $A_{p}$ for all $p \in \pi$.

We now assume that $V$ is an irreducible $A G$-module. We may apply Lemma 1 with $A, G V,[A, G] V, V$ in place of $A, G, M, N$ and conclude that it suffices to find $v \in V$ such that $\operatorname{Orb}_{[A, G]}(v)$ is moved by $A_{p}$ for all $p \in \pi$. By the inductive hypothesis we may then assume that $G=[A, G]$. It follows that $O^{\pi^{\prime}}(A G)=A G$, since otherwise a proper factor group of $G$ would be centralized by $A$, contrary to $G=[A, G]$.

Suppose that $V$ is imprimitive. Let $V=V_{1} \oplus \cdots \oplus V_{t}$ be an imprimitivity decomposition for the action of $A G$ on $V$. We may partition $\{1, \ldots, t\}$ into blocks $B_{j}, 1 \leqslant j \leqslant s$, and set $U_{j}=\sum_{i \in B_{j}} V_{i}$, so that $A G$ permutes the set $\left\{U_{1}, \ldots, U_{s}\right\}$ primitively. Let $C$ be the kernel of the permutation action of $A G$ on the $U_{j}$. Since $A G=O^{\pi^{\prime}}(A G)$, we have $A \nless C$. By [1, Theorem 1] we may choose $S \leqslant\{1,2, \ldots, s\}$ so that the stabilizer in $A G$ of $\sum_{j \in S} U_{j}$ is $C$. Let $U=\sum_{j \in S} U_{j}$. Let $\pi_{1}=\{p \in \pi$ : $\left.A_{p} \leqslant C\right\}$.

Let $A_{1}=\prod_{p \in \pi_{1}} A_{p}$ so that $C=A_{1}(C \cap G)$. All the irreducible constituents of $V_{C \cap G}$ are $A G$-conjugate. Thus if $K_{j}$ denotes the kernel of $C$ on $U_{j}$, then $\cap_{x \in A G} K_{j}^{x}$ $=1$ for each $j \in S$. Let $p \in \pi_{1}$. Since all $p$-Sylow subgroups of $C$ are conjugate under $G \cap C$, it follows that $\left[A_{p}, G \cap C\right] \operatorname{char} G \cap C$, and so $\left[A_{p}, G \cap C\right]^{\prime} \operatorname{char} G \cap$ $C$. The last two sentences imply that $\left[A_{p}, G \cap C\right]^{\prime} \nless K_{j}$ for any $j \in S$. Hence $A_{p} \nless K_{j}$ for $p \in \pi_{1}$ and $j \in S$. Since $K_{j} \triangleleft C$, it follows that $K_{j} \leqslant G \cap C$ and $\left[A_{p},(G \cap C) / K_{j}\right]$ is nonabelian for $p \in \pi_{1}$ and $j \in S$.

For $j \in S$, we may now apply the inductive hypothesis with $A_{1},(G \cap C) / K_{j}, U_{j}$ in place of $A, G, V$. We obtain $u_{j} \in U_{j}$ such that $\operatorname{Orb}_{G \cap C}\left(u_{j}\right)$ is moved by $A_{p}$ for all $p \in \pi_{1}$. Let $u=\sum_{j \in S} u_{j}$. If $p \in \pi-\pi_{1}$ and $P \in \operatorname{Syl}_{p}(A G)$, then the choice of $S$ insures that $P$ does not centralize $u$. If $P \in \operatorname{Syl}_{p}(A G)$ and $p \in \pi_{1}$, then the 
definition of $u_{j}$ implies that $P$ does not centralize $u$. For every $p \in \pi$, the last two sentences show that $A_{p}$ fixes no element in $\operatorname{Orb}_{G}(u)$. If $A_{p}$ stabilized $\operatorname{Orb}_{G}(u)$, then Glauberman's Lemma applied to the action of $A_{p} G$ on $\operatorname{Orb}_{G}(u)$ would yield a contradiction. Hence $A_{p}$ moves $\operatorname{Orb}_{G}(u)$ as desired.

We may now assume that $V$ is a primitive $A G$-module. If $F(A G) \nless G$, then some $A_{p} \leqslant F(A G)$, and so $A_{p} \triangleleft A G$, contrary to $\left[A_{p}, G\right] \neq 1$. Hence $F(A G) \leqslant G$, and so $F(A G)=F(G)$. Set $F(A G)=F$. Now $A G$ can play the role of " $G$ " in Lemma 2 . Let $T, U \leqslant A G$ be as in the conclusion of Lemma 2. Suppose $T \neq U$. Then every 2'-element of $A G$ centralizes $O_{2}(T)$, so $A G / C_{A G}\left(O_{2}(T)\right)$ is a nonidentity 2-group, contradicting $O^{\pi^{\prime}}(A G)=A G$. Thus $T=U$ is cyclic. Let $Z, D$, and $E$ be as in Lemma 2, so that $F=C_{D}(E / Z)$ and each Sylow subgroup of $E / Z$ is a completely reducible $D / F$-module.

Fix $p \in \pi$. Since $A G / D$ is abelian, $\left[A_{p}, G\right] \leqslant D \cap G$. Thus $\left[A_{p}, G\right]=\left[A_{p}, A_{p}, G\right]$ $=\left[A_{p}, D \cap G\right]$. Suppose $\left[A_{p}, E / Z\right]=1$. Since $D$ and $C_{A G}(E / Z)$ are normal in $A G$, we have $\left[A_{p}, G\right]=\left[A_{p}, D \cap G\right] \leqslant C_{D}(E / Z)=F$. Hence

$$
\left[A_{p}, G\right]=\left[A_{p}, A_{p}, G\right]=\left[A_{p}, F\right]=\left[A_{p}, E\right]\left[A_{p}, T\right] \leqslant Z T=T,
$$

contrary to the hypotheses of Proposition 3. Hence $\left[A_{p}, E / Z\right] \neq 1$. Let $E_{1}$ be a Sylow subgroup of $E$ with $\left[A_{p}, E_{1} / E_{1} \cap Z\right] \neq 1$. We apply Lemma 4 to $A_{p} E_{1}, E_{1}, A_{p}, V$ in place of $H, E, P, V$. We conclude that $\left|C_{V}\left(A_{p}\right)\right| \leqslant|V|^{1 / 2}$.

Let $Y$ be an irreducible $F$-submodule of $V$. By Lemma 3, $|Y|=|W|^{\text {me }}$, where $e^{2}=|E: Z|$ and $m$ is a positive integer. Moreover $W$ is a faithful irreducible $T$-submodule of $Y$, so that $|T|$ divides $|W|-1$.

Now $|G \cap D|=|G \cap D: F||F|$. By Lemmas 2 and 5, and an obvious subdirect product argument, $|D: F| \leqslant|E: Z|^{9 / 4}=e^{9 / 2}$. We have $|G \cap D| \leqslant e^{9 / 2} e^{2}|T|=$ $|T| e^{13 / 2}$. Since $\left[A_{p}, G\right]=\left[A_{p}, G \cap D\right]$, we have $O^{p^{\prime}}(A G) \leqslant A_{p}(G \cap D)$, so $\left|\operatorname{Syl}_{p}(A G)\right| \leqslant|G \cap D| \leqslant e^{13 / 2}|T|$. Hence

$$
\sum_{P \in \operatorname{Syl}_{p}(A G)}\left|C_{V}(P)\right| \leqslant|T| e^{13 / 2}\left|C_{V}\left(A_{p}\right)\right| \leqslant|T| e^{13 / 2}|V|^{1 / 2} .
$$

We will show that the following inequality holds:

$$
\sum_{P \in \operatorname{Syl}_{p}(A G)}\left|C_{V}(P)\right| \leqslant p^{-2}|V| .
$$

Suppose (*) is false, so that $p^{2}|T| e^{13 / 2}>|V|^{1 / 2} \geqslant|W|^{e / 2}$.

If $\left[A_{p}, Z\right] \neq 1$, then $p$ divides $\mid$ Aut $Z \mid$ and so $p \mid(s-1)$ for some prime divisor $s$ of $|Z|$. Since $Z=T \cap E$, we have $s \mid e$ and $s \leqslant|T|<|W|$. Since $p>17$ and $p \mid(s-1)$, it follows that $s \geqslant 47$. Since $p<s \leqslant|T|<|W|$, we have $|W|^{3} e^{13 / 2}>$ $|W|^{e / 2}$, so that $e^{13 / 2}>|W|^{(e / 2)-3}>48^{(e / 2)-3}$. Hence $e<20$, contrary to $s \geqslant 47$ and $s \mid e$.

Thus we assume $\left[A_{p}, Z\right]=1$. Let $e=\prod_{i} q_{i}^{n_{i}}$ for distinct primes $q_{i}$. Since $\left[A_{p}, E / Z\right] \neq 1$ and $A_{p} \leqslant D, p$ divides $\left|\operatorname{Sp}\left(2 n_{i}, q_{i}\right)\right|$ for some $i$. Hence $p \mid q_{i}^{2 m_{i}}-1$ for some $m_{i}$ with $1 \leqslant m_{i} \leqslant n_{i}$. Thus $p \mid q_{i}^{m_{i}}+1$ or $p \mid q_{i}^{m_{i}}-1$, where $q_{i}^{m_{i}} \mid e$. It follows that $p \leqslant e+1$. Since $|T|<|W|$, we have $(e+1)^{2} e^{13 / 2}>|W|^{(e / 2)-1}$. Since $|W| \geqslant 3$, we have $e<70$. 
If $m_{i}>1$, our hypothesis that $p \notin \pi_{0}$ implies that $q_{i}^{m_{i}}>70$. Hence $e>70$, a contradiction. Thus $m_{i}=1$ and $p \notin \pi_{0}$ implies that $q_{i} \geqslant 37$. Since $q_{i}$ divides $|T|$ and $|T|$ divides $|W|-1$ and $|W|$ is a prime power, we must have $|W| \geqslant 83$. Hence $(e+1)^{2} e^{13 / 2}>83^{(e / 2)-1}$. As above, this implies that $e<20$, contrary to $q_{i} \mid e$ and $q_{i} \geqslant 37$.

We conclude that $(*)$ holds for each $p \in \pi$. Since $\sum_{p \in \pi} p^{-2}$ is less than 1 , it follows that there exists $v \in V$ such that $v$ is centralized by no $p$-Sylow subgroup of $A G$ for any $p \in \pi$. Then no $A_{p}$ fixes any element in $\operatorname{Orb}_{G}(v)$. By Glauberman's Lemma, each $A_{p}$ moves $\operatorname{Orb}_{G}(v)$.

Proposition 4. Let $A$ be cyclic of squarefree $\pi_{0}^{\prime}$-order. Let $N$ be an $A$-invariant normal abelian subgroup of $G$ which is a direct product of completely reducible $A G$-modules. Suppose $N=C_{A G}(N)$. For each $p \in \pi(A)$, suppose that $\left[A_{p}, G / N\right]$ is either nonabelian or trivial. Then $A$ has a faithful orbit on $\operatorname{Irr}(G)$.

Proof. Let $V=\operatorname{Irr}(N), \pi=\pi(A), \pi_{1}=\left\{p \in \pi:\left[A_{p}, G / N\right]\right.$ is nonabelian $\}$, and $\pi_{2}=\pi-\pi_{1}$. Let $A_{1}$ be the Hall $\pi_{1}$-subgroup of $A$.

Then $A_{1}, G / N$, and $N$ satisfy the hypotheses of Proposition 3. Hence we may choose $v \in V$ so that $\operatorname{Orb}_{G}(v)$ is moved by $A_{p}$ for all $p \in \pi_{1}$.

Write $V=V_{1} \times \cdots \times V_{k}$, a direct product of irreducible $A G$-modules. We may assume that each component $v_{i}$ of $v$ is not 1 . For each $p \in \pi_{2}$, we may choose $i \in\{1, \ldots, k\}$ such that $V_{i}$ is not centralized by $A_{p}$. Since $\left[A_{p}, G\right] \leqslant N=C_{A G}(V)$, the centralizer in $V_{i}$ of $A_{p}$ is an $A G$-submodule of $V_{i}$, and hence is trivial. Thus $A_{p}$ moves $v_{i}$, and so $A_{p}$ moves $v$.

We think of $v$ as a linear character $\lambda$ of $N$. Then $\operatorname{Orb}_{N}(\lambda)=\{\lambda\}$ is not $A_{p}$-invariant for any $p \in \pi_{2}$. By Lemma 1 with $A, G, N, N$ in place of $A, G, M, N$, we conclude that $\operatorname{Orb}_{G}(\lambda)$ is moved by $A_{p}$ for all $p \in \pi_{2}$. By the second paragraph, $\operatorname{Orb}_{G}(\lambda)$ is moved by $A_{p}$ for all $p \in \pi$. Hence if $\chi \in \operatorname{Irr}(G \mid \lambda)$, then $\chi$ lies in a faithful $A$-orbit.

Proof of Theorem 1. We may assume $A$ is cyclic of squarefree order. The hypotheses of Theorem 1 imply that $F(A G)=F(G)$. Since $G=\left[A_{p}, G\right] C_{G}\left(A_{p}\right)$ for each $p \in \pi(A)$, it follows that $A$ acts faithfully on $G / \Phi(A G)$. Thus we may assume that $\Phi(A G)=1$ and hence that $F(G)=F(A G)$ is a direct product of completely reducible $A G$-modules (see [3, III, Satz 4.5]).

Partition $\pi(A)=\pi$ as follows. Let $\pi_{1}=\pi \cap \pi_{0}, \pi_{2}=\left\{p \in \pi: p \notin \pi_{0}\right.$ and $\left[A_{p}, G / F(G)\right]$ is abelian and nontrivial $\}, \pi_{3}=\pi-\pi_{1}-\pi_{2}$, and $\pi_{4}$ be the larger of $\pi_{2}$ and $\pi_{3}$. Let $A_{4}$ be the Hall $\pi_{4}$-subgroup of $A$. By Proposition 3 or Proposition 4 applied to $A_{4}$ and $G$, we may conclude that $A_{4}$ has a faithful orbit on $\operatorname{Irr}(G)$. Since $|\pi(A)| \leqslant 2\left|\pi\left(A_{4}\right)\right|+8$, this completes the proof.

\section{REFERENCES}

1. D. Gluck, Trivial set-stabilizers in finite permutation groups, Canad. J. Math. 35 (1983), 59-67.

2. D. Gluck and T. R. Wolf, Defect groups and character heights in blocks of solvable groups. II, J. Algebra 87 (1984), 222-246. 
3. B. Huppert, Endliche Gruppen, Springer-Verlag, Berlin, 1967.

4. I. M. Isaacs, Character theory of finite groups, Academic Press, New York, 1976.

5. __ Solvable group character degrees and sets of primes, J. Algebra 104 (1986), 209-230.

6. T. R. Wolf, Defect groups and character heights in blocks of solvable groups, J. Algebra 72 (1981), 183-209.

7. __ Solvable and nilpotent subgroups of $\mathrm{GL}\left(n, q^{m}\right)$, Canad. J. Math. 34 (1982), 1097-1111.

Department of Mathematics, Wayne State University, Detroit, Michigan 48202 